\title{
Origin of cerebrospinal fluid melatonin and possible function in the integration of photoperiod
}

\author{
H. Tricoire ${ }^{1}$, M. Møller ${ }^{2}$, P. Chemineau ${ }^{1}$ and B. Malpaux ${ }^{1 *}$ \\ ${ }^{1}$ UMR INRA-CNRS-Université de Tours, Physiologie de la Reproduction et des Comportements, \\ 37380 Nouzilly, France: and ${ }^{2}$ Institute of Medical Anatomy, University of Copenhagen, Panum \\ Institute, Copenhagen DK-2200, Denmark
}

\begin{abstract}
Melatonin, which is synthesized at night by the pineal gland, is present in the cerebrospinal fluid (CSF), but its entry site and its role in this compartment are not known. Using several approaches, we tested the hypothesis that melatonin enters the CSF through the pineal recess, an evagination of the third ventricle. CSF melatonin concentrations are higher near the pineal gland than in the anterior part of the third ventricle, and decrease markedly $(80 \%)$ after sealing off the pineal recess. Moreover, ultrastructure and permeability analyses of the pineal-CSF interface showed that melatonin could reach the CSF either via delivery in situ by protruding pinealocytes that make direct contact with the CSF or via extracellular secretion and interstitial fluid draining into the ventricular lumen. These data indicate that melatonin in the CSF probably originates from a few pinealocytes of the basal part of the pineal gland neighbouring the pineal recess. Melatonin carried to the brain by the blood appears to be able to mediate the effects of photoperiod on reproduction, but it is unclear whether melatonin in CSF may fine-tune this response both in terms of timing and amplitude. It is critical to determine which pathway, blood or CSF, allows melatonin to reach its central targets more efficiently.
\end{abstract}

\section{Introduction}

Among the various functions of melatonin, a well-characterized role of this indolamine is the transduction of photoperiodic information to control reproduction (Arendt, 1995). The control of reproduction occurs as a result of regulatory effects on pulsatile GnRH secretion of changes in the daily rhythm of melatonin synthesis in the pineal gland, that result in high concentrations at night and low concentrations in the day. For instance, in sheep, long periods of melatonin secretion, as seen during short days, are stimulatory to the GnRH system. Melatonin acts on receptors located in the pre-mammillary hypothalamus to produce this effect and is thought to be transported in the blood to this target. However, melatonin could also be transported to this target area by the cerebrospinal fluid (CSF). The proposal that melatonin is transported to the target area by CSF has recently regained interest because there 
is accumulating evidence for a role of CSF in neural communication (Lehman and Silver, 2000) and because melatonin concentrations in the third ventricle are at least 20 -fold higher than in the blood (Skinner and Malpaux, 1999). The present review outlines the evidence for a role of CSF melatonin in the regulation of reproductive function. After a brief review of the characteristics of CSF, the data demonstrating how melatonin reaches the CSF will be presented. Subsequently, the possible role of CSF melatonin in the integration of photoperiod will be analysed.

\section{CSF and neural communication}

The CSF is a clear colourless solution contained in the subarachnoidal space and the ventricular system of brain and spinal cord. The ventricular system includes four CSF-filled cavities known as ventricles (paired lateral, third and fourth), which communicate with each other and with the subarachnoidal space. The lateral cerebral ventricular walls and surfaces of non-circumventricular organs are composed of ciliated cuboidal ependymal cells, which can secrete CSF occasionally, but are mainly involved in the local CSF flow and circulation by the movement of their cilia (Scott et al., 1982). Except in the circumventricular organs, ependymal cells are not linked by tight junctions, indicating that the interstitial fluid within the brain communicates freely with the CSF.

Under normal conditions, the choroid plexus present within each of the four cerebral ventricles produces CSF; the choroid plexus of the lateral ventricles is the main source. The choroid plexus consists of a single continuous layer of epithelial cells that are bound by tight junctions, overlying a vascular central core devoid of a blood-brain barrier. The epithelial cells are the component of the choroid plexus that secretes CSF by a process involving the movement of $\mathrm{Na}^{+}, \mathrm{Cl}^{-}$and $\mathrm{HCO}_{3}{ }^{-}$from the blood to the ventricles of the brain: this transfer creates the osmotic gradient that drives the secretion of $\mathrm{H}_{2} \mathrm{O}$ (for a review, see Speake et al, 2001). The production rate of CSF in humans is approximately $400 \mu / \mathrm{min}^{-1}$, and the total volume is $80-150 \mathrm{ml}$ (McComb, 1983), whereas in sheep the production rate is estimated at about $120 \mu \mathrm{l} \mathrm{min}{ }^{-1}$ for a total volume of $15 \mathrm{ml}$ (Evans et al., 1974).

Although its composition is very similar to that of plasma, except for greatly reduced protein content, CSF is not an ultrafiltrate of plasma. The CSF contains molecules originating in blood, or produced and delivered in situ by cells that make contact with CSF, or diffuse from the extracellular space. Many active substances are detected in the CSF, namely, hypothalamic releasing hormones (thyrotrophin releasing hormone $(\mathrm{TRH}), \mathrm{GnRH}$, somatostatin, corticotrophin releasing hormone $(\mathrm{RH})$ ), binding proteins (transthyretin, prostaglandin D synthase, albumin), pituitary hormones (thyroid-stimulating hormone (TSH), LH, FSH, prolactin, growth hormone, antidiuretic hormone $(\mathrm{ADH})$ ), steroid and pancreatic hormones (testosterone, oestradiol, progesterone, insulin), neuropeptides (opioids, cholecystokinin (CCK), vasoactive intestinal peptide (VIP)), neuroendocrine hormones (serotonin, melatonin) and cytokines (Wood, 1982). It is worth noting that the composition of CSF along its circulation system is not uniform: each cavity is delimited by specific cerebral structures capable of modifying the local concentrations of the molecules present (active uptake, dilution, catabolism, secretion) (Strikic et al., 1994).

Several functions have been attributed to CSF. CSF fills the ventricles and surrounds the external surfaces of the brain, acting both as a fluid cushion and as a drainage route for the products of cerebral metabolism. It is also believed to be nutritive for both neurones and glial cells, and to maintain the constant ionic composition of the local microenvironment of the brain cells (Nilsson et al., 1992). Nicholson (1999) suggested that CSF may act to 
convey substances that are present within the CSF or secreted into the CSF rapidly to many brain regions. This hypothesis parallels the 'volume transmission' concept, which indicates that intercellular communication in the brain can occur via a three-dimensional diffusion of a signal in the extracellular fluid volume for a distance greater than the synaptic cleft (Zoli et al., 1998). This concept is supported by mismatches between neurotransmitter and receptor localization in the brain (Herkenham, 1987).

The basic elements of volume transmission are a cell source that releases a diffusible signal, which is transported by a communication channel, the extracellular fluid, in order to reach a target cell in the brain. Considering CSF as a transport medium for chemical signals requires the demonstration that these basic elements in the volume transmission are present in the ventricular system, using the CSF, and not the extracellular fluid, as the communication channel. The source of CSF molecules may vary: blood (choroid plexus, cerebral capillaries), cells that make contact with CSF (ependymal cells, supra-ependymal neurones, epithelial cells of choroid plexu) and extracellular fluid of the cerebral tissue close to the ventricular system. It has been demonstrated using autoradiography after injection of $\left[{ }^{14} \mathrm{C}\right]$ inulin into the lateral ventricle that molecules present within the CSF can reach virtually all regions of the brain (Proescholdt et al., 2000). Although there was preferential penetration of the labelled inulin into the hypothalamus and brainstem, virtually the entire brain was labelled after $4 \mathrm{~h}$, and it is possible that this phenomenon could be faster for smaller molecules.

There are many examples of the induction of physiological effects after intracerebroventricular injection of molecules that are found naturally within the CSF. However, these examples do not demonstrate that CSF acts as a transport medium for these molecules as many of the concentrations induced by these injections are not necessarily physiological. Nevertheless, evidence has been obtained that would support the concept that CSF is the physiological transport medium for some molecules. For example, in hamsters with lesions of the suprachiasmatic nucleus, circadian activity rhythms can be sustained by means of a diffusible signal originating in suprachiasmatic nucleus grafts, which could not display neural outgrowth (Silver et al., 1996). There is also evidence for the presence of an endogenous anticonvulsant substance in rat cerebrospinal fluid, possibly opioid in nature, which is activated as a consequence of a seizure and may play a critical role in post-seizure inhibition (Tortella and Long, 1985). In addition, it was demonstrated that CSF taken from sleep-deprived goats was able to increase the percentage of non-REM sleep in rats, indicating that CSF may have a role as a sleep signal (Pappenheimer et al., 1967). The identity of these CSF signals is unknown, except perhaps in the case of the sleep signal in which CSF concentrations of interleukin $1 \beta$ have been shown to vary with sleep and sleep deprivation (Krueger et al., 1998). GnRH is another candidate molecule that may use CSF as transport medium as it has been shown that the concentrations of GnRH in the CSF can change simultaneously with those in the blood, and $\mathrm{GnRH}$ axons penetrate the ventricular ependyma, indicating that $\mathrm{GnRH}$ might be secreted directly into CSF. Although the function of GnRH in CSF remains unknown, it may play an important role in sexual behaviour and GnRH in CSF could be involved in this function (Skinner and Caraty, 2002).

\section{Characteristics and origin of CSF melatonin}

Melatonin can be detected in the CSF of many species. Nocturnal CSF melatonin concentrations in cisterna magna are approximately $30 \mathrm{pg} \mathrm{ml}^{-1}$ in rats (Withyachumnarnkul and Knigge, 1980), $10-300 \mathrm{pg} \mathrm{ml}^{-1}$ in sheep (Rollag et al., 1978) and $20-40 \mathrm{pg} \mathrm{ml}^{-1}$ in rhesus monkeys (Perlow et al., 1981). In the lateral ventricle, CSF melatonin concentrations are much higher at 600-2400 $\mathrm{pg} \mathrm{ml}^{-1}$ in sheep, goats and cows (Hedlund et al., 1977; Kanematsu et al., 1989; 
Shaw et al., 1989; Skinner and Malpaux, 1999) and even higher in the third ventricle in sheep at a ratio of $1: 7$ between lateral and third ventricle (Skinner and Malpaux, 1999). In contrast, the concentrations of melatonin measured in CSF that was collected by lumbar puncture in humans and thesus monkeys are much lower than ventricular concentrations and are similar to the cisterna magna concentrations (20-90 $\mathrm{pg} \mathrm{ml}^{-1}$ and $10-40 \mathrm{pg} \mathrm{ml}^{-1}$, respectively) (Bruce et al., 1991; Reppert et al., 1979).

Melatonin concentrations in CSF display a day-night rhythm in various species independent of the CSF collection site. The nocturnal increase in CSF melatonin concentrations is twofold when measured in the cisterna magna of rats (Withyachumnarnkul and Knigge, 1980), 5- to 20-fold in lumbar CSF of humans and rhesus monkeys (Reppert et al., 1979; Bruce et al., 1991), 12- to 17-fold in the lateral ventricle of goats and cows (Hedlund et al., 1977; Kanematsu et al., 1989) and 35-fold in the third ventricle of sheep (Skinner and Malpaux, 1999). In sheep, the dusk increase and dawn decrease in melatonin concentrations are as marked in both blood and third ventricle CSF and, thus, the coding for duration of melatonin is equally good in both compartments. However, CSF melatonin concentrations are 20-fold higher than the blood concentrations (Skinner and Malpaux, 1999). One key question is how such a difference in the concentrations of melatonin can be obtained between these two liquid compartments, CSF versus blood. Several hypotheses have been proposed to explain the relatively high concentrations of melatonin in CSF. The first hypothesis is that there is active uptake of melatonin from peripheral blood and the second is that there is release of melatonin from the choroid plexus after retrograde transport from the Galen vein (Skinner and Malpaux, 1999). However, neither of these hypotheses have received convincing experimental support. The third ventricle which contains CSF extends into the pineal gland by means of the pineal recess, which separates two laminae forming the stalk and attaching the epiphysis to the brain (Vollrath, 1981). Several different forms of evidence indicate that the pineal recess is the main site through which melatonin enters the CSF. Namely, melatonin concentrations are considerably higher within the pineal recess than in the ventral part of the third ventricle, despite separation by as little as $10 \mathrm{~mm}$. Withdrawal of CSF from the pineal recess causes a flow rate-dependent decrease in melatonin concentration in the rest of the third ventricle (Tricoire et al., 2002). Surgical obliteration of the pineal recess which prevents the exchange of CSF between the recess and the rest of the ventricle causes a decrease of about $80 \%$ in melatonin concentrations in the third ventricle (Fig. 1). All of these data demonstrate that most of the melatonin in CSF enters the third ventricle through the pineal recess, and from there, diffuses to the whole third ventricle. This conclusion is reinforced by the morphology of the cells bordering the pineal recess of sheep (Fig. 2). Indeed, the ependymal lining of the third ventricle was no longer apparent in the part of the pineal recess that apposes the basal part of the pineal gland of the sheep. In this area, pinealocytes are directly apposed to the CSF, often bulging into the ventricular lumen of the pineal recess (Tricoire et al., 2003). In addition, the area of pineal-CSF contact is also quite large compared with that of rodents and primates, and the pineal gland-CSF interface is highly permeable to molecules, such as horseradish peroxidase, with a molecular mass of at least $40 \mathrm{kDa}$. Therefore, molecules newly synthesized within the pinealocytes, for example melatonin, could reach the CSF by two non-exclusive mechanisms: (i) direct release into the CSF from protruding cells that make direct contact with the CSF, or (ii) release into the pineal interstitial fluid that subsequently drains into the ventricular lumen (Tricoire et al., 2003). The identification of this specialized site of entrance of melatonin into the CSF raises two major points.

First, it strongly indicates that melatonin in CSF originates from pinealocytes located in the basal part of the pineal gland, that is next to the CSF, whereas melatonin that is found in the peripheral circulation comes from pinealocytes neighbouring blood vessels throughout 
(a)

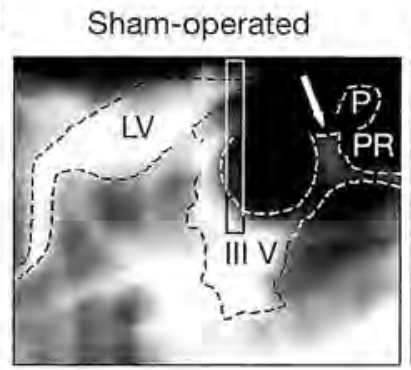

Failed to seal off

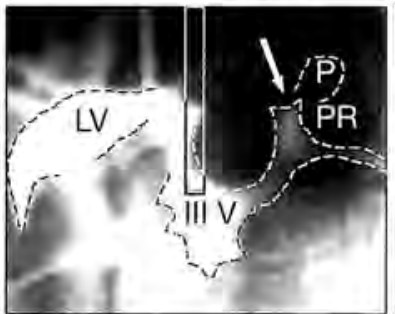

Sealed off

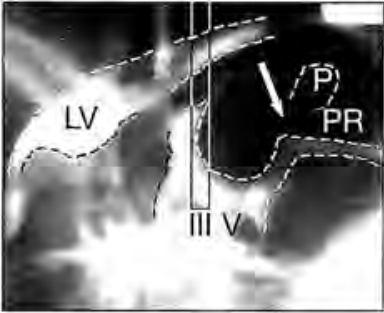

(b)

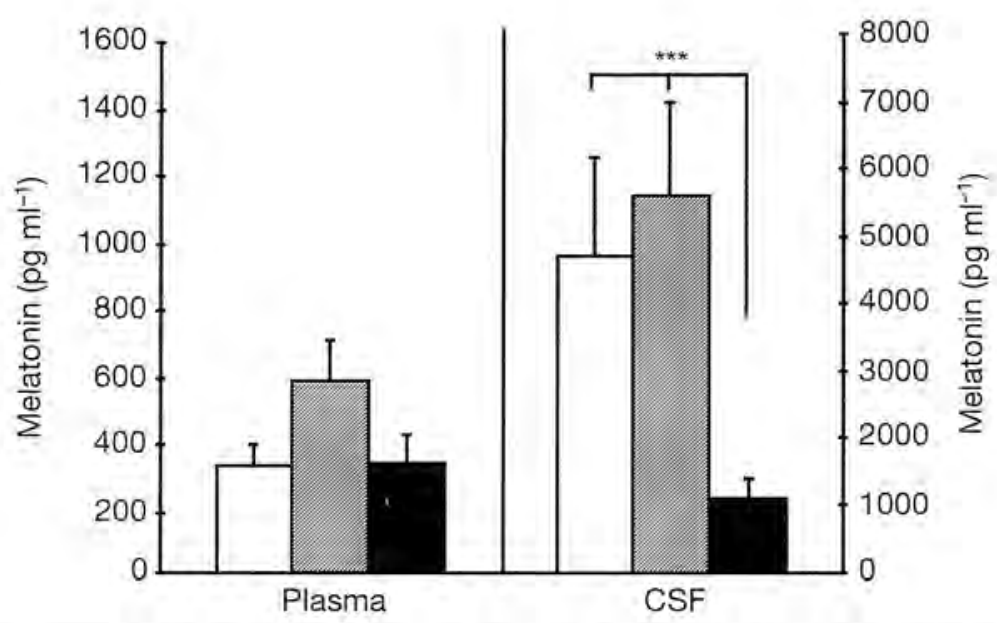

Fig. 1. (a) X-rays of the ventricular system used to determine the completeness of the surgical sealing off of the pineal recess (PR) (radio-opaque fills or does not fill the PR) in sheep. The vertical bar is the guide cannula for collection of cerebrospinal fluid (CSF), LV: lateral ventricle; P: pineal gland; III V: third ventricle. The arrows point to PR. (b) Effects of sealing off the PR on melatonin concentrations in jugular blood and in the CSF of the third ventricle. $\square$, sham-operated; $\square$, failed to seal off; $\mathbf{\square}$, sealed off. $* * * P<0.005$. (Adapted from Tricoire et al., 2002a.)

the pineal gland. This functional division of the ovine pineal gland (basal part producing CSF melatonin, remainder of the pineal producing blood melatonin) may be compared with the division of the pineal gland of rodents into a superficial pineal and a deep pineal; only the deep pineal is in contact with the pineal recess (Møller, 1981), It is possible that in these species, the deep pineal gland releases its secretory products mainly into the CSF, whereas the superficial pineal gland secretes melatonin into the blood.

Second, melatonin must progress against the dominant rostro-caudal flow to reach the ventral part of the third ventricle. Indeed, the commonly accepted model for CSF circulation assumes a bulk flow, that is a consistent net flow of CSF, from the choroid plexus in the ventricular system to the pacchionian granulations (arachnoid villi) where CSF outflow takes place. However, this concept has been challenged by recent observations using flow-sensitive magnetic resonance imaging and radionuclide cisternography (Greitz and Hannerz, 1996). After tracers are administered into the subarachnoid space, CSF and its constituents are not exclusively drained through the pacchionian granulations until the venous sinus, but seem to be absorbed into the capillaries of the central nervous system by the communication between 

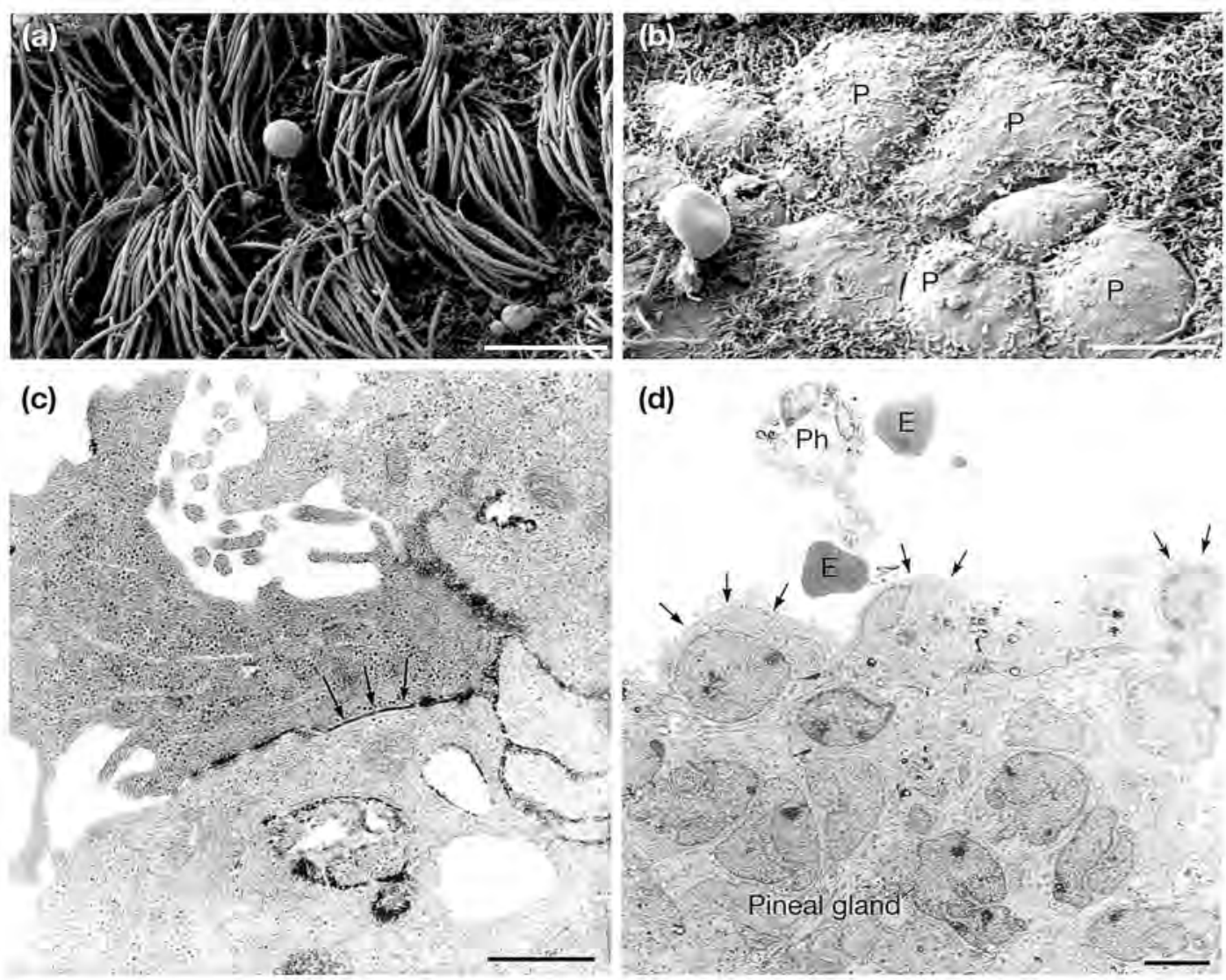

Fig. 2. Scanning electron micrographs of (a) the ciliated lateral parts of the pineal recess of sheep, and (b) the bulging pinealocytes (P) in the central part of the pineal recess bordering the pineal gland of sheep. Note that the number of cilia markedly decreases and that the pinealocytes directly contact the cerebrospinal fluid. (c) Electron micrograph of the luminal layer of pinealocytes bordering the pineal recess of sheep. Horseradish peroxidase, which has been injected into the third ventricle, is seen to permeate the ventricular junctional complexes, consisting of a gap junction (arrows) bordered on each site of intermediate junctions. (d) Electron micrograph of the border between the pineal recess and the pineal gland of the sheep. The pinealocytes are in direct contact with the cerebrospinal fluid and several of the pinealocytes (arrows) are protruding into the ventricular lumen. E: erythrocyte. Ph: intraventricular phagocyte. Scale bars represent $(a, b)$ $5 \mu \mathrm{m}$, (c) $2 \mu \mathrm{m}$ and (d) $7 \mu \mathrm{m}$.

CSF, extracellular space and brain capillaries. In addition, CSF circulation does not follow a unique rostro-caudal bulk flow, but undergoes pulsations synchronous with the cardiac cycle and is affected by variations in venous pressure. When the CSF circulation velocity is high (in aqueduct and foramina), a caudal net flow is observed; however, a lower velocity in larger cavities or spaces leads to circular motion (in the third ventricle) or multi-directional flow (Henry-Feugeas et al., 1993).

\section{Possible function of CSF melatonin in the photoperiodic control of reproduction}

The demonstration that the pineal gland discharges melatonin directly into the CSF of the third ventricle and the subsequent presence of high concentrations of the indolamine in this 
compartment raise the question of the possible function of this specialized delivery mechanism. We therefore assessed whether CSF melatonin played a role in mediating the effects of photoperiod on reproductive activity in sheep. It has previously been assumed that these effects are mediated by blood-borne melatonin for two main reasons. First, most of the melatonin synthesized by the pineal gland is secreted into the peripheral circulation, whereas only a small proportion, $<1 \%$, is released into the CSF (Rollag et al., 1978). Nevertheless, as pointed out above, this small amount is sufficient to increase CSF concentrations of the indolamine because of the small distribution volume compared with blood ( $15 \mathrm{ml}$ versus 5 I of blood in sheep). Second, melatonin administered systemically to pinealectomized animals can mimic the effects of photoperiod in pineal-intact animals (Bittman et al., 1983). However, these data must be interpreted with caution in terms of the route used by melatonin to get from its source to its target. Indeed, the concentralions produced in these replacement studies replicated jugular concentrations and not carotid artery concentrations, which are several-fold lower (B. Malpaux, L. Zarazaga, L. Todini, D. C. Skinner, P. Chemineau, unpublished). The blood signal reaching the brain was therefore supra-physiological in these studies. Furthermore, the initial removal of melatonin by pinealectomy may modify the sensitivity of the target sites to melatonin as indicated by an increase in the density of melatonin receptors in the pars tuberalis in pinealectomized animals (Schuster et al., 2001). The animal could be subsequently responsive to melatonin concentrations that would normally be insufficient. These last studies showed unequivocally that melatonin was the primary transducer of photoperiodic information, but it now appears that no extrapolation about the route used by melatonin to get from its source to its target is possible.

The possible role of melatonin in CSF must now be considered in light of four main pieces of evidence. First, the binding sites of melatonin involved in transducing its effect on reproduction are located in a periventricular position. In ewes, the presence of binding sites for melatonin has been documented in the pre-mammillary hypothalamus and the local delivery of melatonin in this site is sufficient to stimulate $\mathrm{LH}$ secretion in the same way as a large amount of melatonin administered peripherally (Malpaux et al., 1998). All of these binding sites are located $<2 \mathrm{~mm}$ from the lumen of the third ventricle. Second, as pointed out above, CSF melatonin concentrations in the third ventricle, that is, in the vicinity of melatonin targets, are at least 20 times higher than blood melatonin concentrations. Third, melatonin is a small molecule ( $\mathrm{MW}=232 \mathrm{~g} \mathrm{~mole}^{-1}$ ) that is highly lipophilic and, therefore, should diffuse easily in the brain tissue. The diffusion of melatonin has no been extensively studied, but after a bolus injection into the lateral ventricle of rats, melatonin is found in large amounts in the hypothalamus (Anton-Tay and Wurtman, 1969). In addition, the disappearance of melatonin as indicated by the low concentration of melatonin in the CSF (same concentration as in blood) when it is collected by lumbar puncture indicates that it diffuses into the surrounding tissues. Fourth, melatonin is present in the CSF throughout the night and this continuous supply of the hormone to the brain tissue is favourable to a deeper diffusion.

An experimental model was developed to test whether the specific removal of CSF melatonin would alter the response to photoperiod and was subsequently used to test the hypothesis that CSF melatonin is the main transducer of photoperiodic information to the reproductive axis. In a preliminary experiment, the removal of the long-day inhibitory signal by pinealectomy leading to stimulation of reproductive neuroendocrine activity was examined. Ovariectomized ewes treated with a s.c. implant of oestradiol were exposed to inhibitory long days ( $16 \mathrm{~h}$ light: $8 \mathrm{~h}$ dark). Between day 75 and day 85 of exposure to long days, ewes were pinealectomized. As a consequence, $\mathrm{LH}$ secretion increased in these ewes on average at day 61 after the surgery. In contrast, LH secretion remained inhibited for at least 90 days in the control animals. 
If melatonin in CSF transduces photoperiodic information, the sealing off of the pineal recess leading to a large decrease in melatonin concentrations in CSF without affecting blood concentrations should produce the same effect as pinealectomy on $\mathrm{LH}$ secretion of ewes exposed to long days, that is an increase in LH secretion after 50-60 days. Animals were initially exposed to 90 short days ( $8 \mathrm{~h}$ light: $16 \mathrm{~h}$ dark) and then to inhibitory long days ( $16 \mathrm{~h}$ light: $8 \mathrm{~h}$ dark) until the end of the experiment. Between day 70 and day 90 of exposure to long days, ewes underwent surgery to seal off the pineal recess and prevent the passage of melatonin from the pineal gland to the CSF (the same animals were used by Tricoire et al., 2002). The effectiveness of the surgery was verified using a lateral X-ray of the head after injection of radio-opaque fluid in the ventricle. The absence of radio-opaque fluid in the pineal recess was interpreted as complete sealing off of the pineal recess. The sealing off of the pineal recess did not result in changes in blood melatonin concentrations relative to shamoperated animals and, therefore, the potential signal carried by the blood was unchanged, but produced an $80 \%$ reduction in melatonin concentrations in CSF (Tricoire et al., 2002). In all animals, whether the pineal recess was sealed off or failed to be sealed off and in shamoperated controls, LH secretion remained inhibited beyond the 50-60 days when an increase would have been expected after the removal of the long-day inhibition (Fig. 3). However, the three groups differed later when they became spontaneously refractory to long days. In the control animals, the increase in LH secretion, resulting from the development of refractoriness to long days was observed as expected after about 6 months of exposure to long days $(187 \pm 6$ and $184 \pm 7$ days in the sham-operated animals and in animals in which the pineal recess had failed to be sealed off, respectively, Fig. 3). In contrast, in animals in which the pineal recess was sealed off, the expression of refractoriness was delayed ( $216 \pm 8$ days, $P<0.05$ relative to both control groups) (Fig. 3). These results indicate that melatonin in the blood was sufficient to convey the long-day signal to the reproductive axis, as the strong reduction in melatonin concentrations in CSF did not result in the removal of the inhibitory signal in the same way as pinealectomy. However, these results must be interpreted with caution for several reasons. First, CSF melatonin was not completely suppressed in the experimental animals. Although CSF melatonin was reduced by about $80 \%$, the remaining concentrations were about $1000 \mathrm{pg} \mathrm{ml}^{-1}$, which is still much higher than carotid blood concentrations (not measured in this experiment, but generally about $100 \mathrm{pg} \mathrm{m}^{-1}$ ). Second, the delay in the development of refractoriness to long days is intriguing. This finding indicates that the reduction of CSF concentrations modified the way in which the animals perceived the inhibitory photoperiod and, therefore, the timing of photorefractoriness. The simplest explanation is that the timing of photorefractoriness is related to the 'strength' of the melatonin signal; the decrease in melatonin concentrations would have led to a reduction in the signal. To our knowledge, there is no evidence to support this possibility. Alternatively, it is possible that after the sealing off of the pineal recess, the melatonin signal initially became insufficient to provide a long-day signal. However, the reduction in melatonin concentrations in CSF modified the sensitivity of the melatonin responsive elements in such a way that the remaining melatonin signal recovers its ability to transduce long day information. The loss of long-day information would not have been for long enough to allow a stimulation of $\mathrm{LH}$ secretion but would have disturbed the timing of long-day refractoriness. This possibility is supported by the demonstration that pinealectomy leads to a large increase in the density of melatonin receptor in the pars tuberalis of hamsters (Schuster et al., 2001) and, more generally, melatonin concentration influences affinity and density of melatonin receptors (Barrett et al., 1996),

In conclusion, the results obtained from the model in which the pineal recess was sealed off indicate that melatonin in CSF is not critical to the interpretation of daylength. However, this model presents several limitations, in particular the remaining presence of melatonin in 
(a)

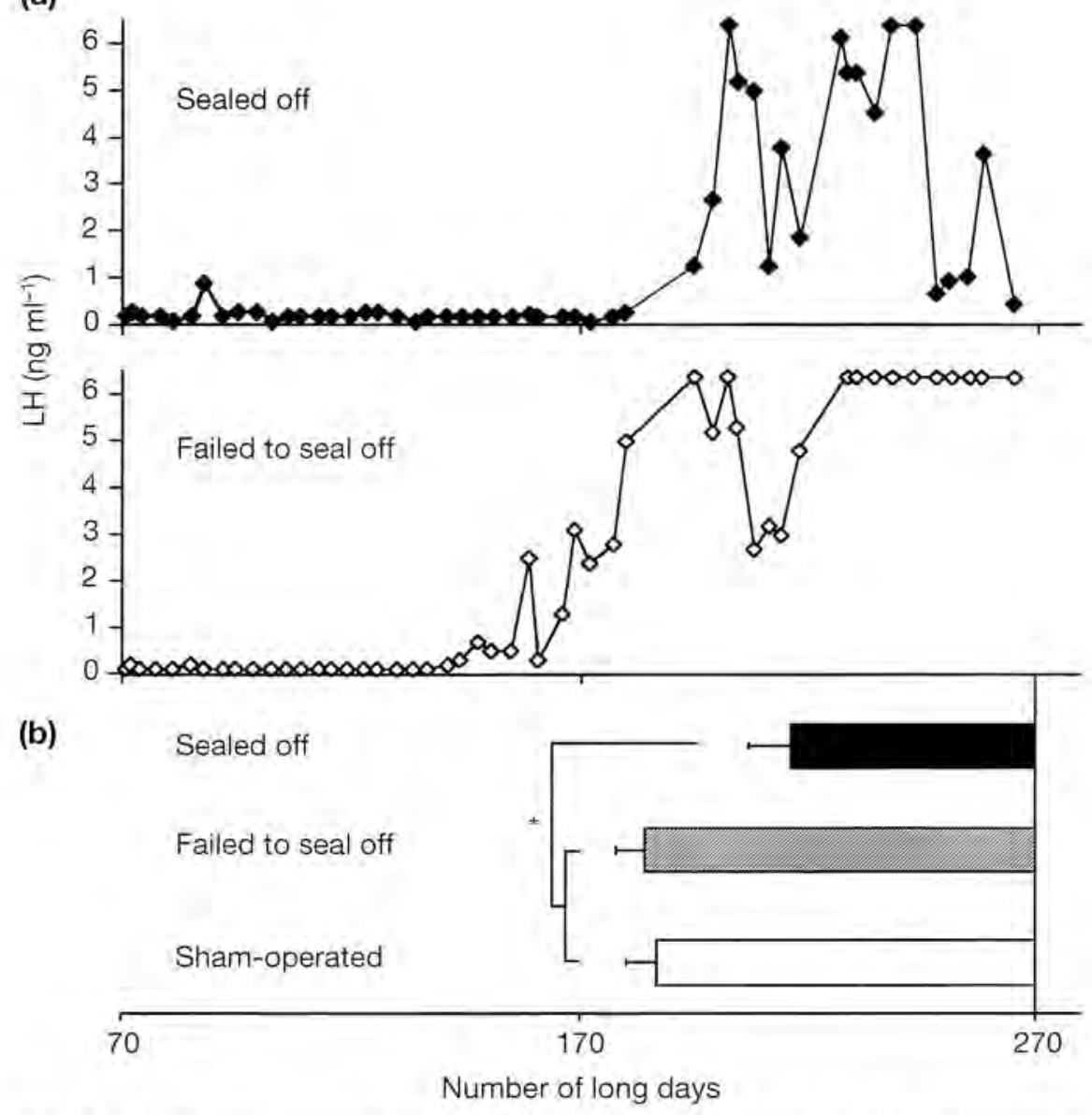

Fig. 3. (a) Plasma concentrations of LH in ovariectomized ewes treated with a continuous release implant of oestradiol. Animals were exposed to long days $(16 \mathrm{~h}$ light: $8 \mathrm{~h}$ dark) and underwent either a surgical sealing off or a failed sealing off of the pineal recess aiter 70 long days, (b) Mean \pm SEM day of increase in LH concentrations for the three groups of ewes ( sealed off, $n=8$; $\square$, failed to seal off, $n=4 ; \square$, sham-operated, $n=6$ ). The day of increase was the first day on which a series of at least three $\mathrm{LH}$ concentrations exceeded $1 \mathrm{ng} \mathrm{ml}^{-1}$.

the CSF and the absence of the control of the sensitivity of the melatonin responsive elements, which make it unsuitable to reach a definitive conclusion. In an attempt to overcome these limitations, another experimental model was developed. Animals are exposed to long days and receive an infusion of melatonin for $8 \mathrm{~h}$ before the night at various concentrations. If melatonin is present in concentrations sufficient to be integrated during the infusion, the summation of this period of exogenous signal and the endogenous secretion of melatonin will provide a short-day signal (long presence of melatonin). In contrast, if the exogenous melatonin is not sufficient to be integrated, the animal will respond to a long-day signal (short presence of endogenous melatonin only). Therefore, this model should allow us to test which concentration of melatonin is required to produce a photoperiodic response. In contrast to the pinealectomized model (Bittman et al., 1983), the presence of endogenous 
concentration of melatonin for $8 \mathrm{~h}$ every day should prevent the changes in the sensitivity of the melatonin-responsive elements. In this model, preliminary data indicate that melatonin delivered to mimic carotid concentrations of melatonin are sufficient to provide a short-day signal but higher concentrations are necessary to obtain a normal timing of the response.

\section{Conclusion}

Melatonin reaches the CSF very efficiently because of the complete disappearance of the ependymal lining of the third ventricle in the part of the pineal recess apposing the pineal gland. In this area, pinealocytes are in direct contact with the CSF, often bulging into the ventricular lumen of the pineal recess, and the permeability of molecules from the CSF into the pineal parenchyma has been demonstrated. Despite the fact that a limited number of pinealocytes are probably concerned, high CSF concentrations of melatonin are still produced, probably due to its limited distribution volume. The respective physiological roles of this CSF melatonin relative to melatonin carried in the blood are difficult to establish, as the manipulation of melatonin concentration in one compartment may interfere with the threshold of sensitivity of the melatonin responsive elements. Although it appears that melatonin transported to the brain by the blood can produce a photoperiodic response, it is unclear whether melatonin in CSF may fine-tune this response both in terms of timing and amplitude. It also remains to be determined whether the diffusion of melatonin from the CSF into the hypothalamus is sufficient to raise tissue contents to higher values than with melatonin from blood alone. In this case, in a normal animal in which hypothalamic melatonin comes from both transport media, blood and CSF, the CSF signal would 'dominate' the blood signal.

H. Tricoire was supported by a grant from 'Région Centre'. The authors wish to thank U. Rentzmann, M-A. Gleie, A. Daveau, S. Canepa, C. Gauthier, A. Locatelli and C. Moussu for their technical assistance.

\section{References}

Anton-Tay F and Wurtman RJ (1969) Regional uptake of $3 \mathrm{H}$-melatonin from blood or cerebrospinal fluid by the rat brain Nature $221474-475$

Arendt I (1995) Melatonin and the Mammalian Pineal Gland Chapman \& Hall, London

Barrell P, MacLean A, Davidson $G$ and Morgan PJ (1996) Regulation of the Mel la melatonin receptor mRNA and protein levels in the ovine pars tuberalis: evidence for a cyclic adenosine $3^{\prime}, 5^{\prime}$ monophosphate-independent Mel ta receptor coupling and an autoregulatory mechanism of expression Molecular Endocrinology 10 892-902

Bittman EL, Dempsey RJ and Karsch FJ (1983) Pineal melatonin secretion drives the reproductive response to daylength in the ewe Endocrinology $1132276-$ 2283

Bruce J, Tamarkin L, Riedel C, Markey S and Oldfield E (1991) Sequential cerebrospinal fluid and plasma sampling in humans: 24-hour melatonin measurements in normal subjects and after peripheral sympathectomy Journal of Clinical Endocrinology and Metabolism 72 819-823

Evans CA, Reynolds JM, Reynolds ML, Saunders NR and Segal MB (1974) The development of a blood- brain barrier mechanism in foetal sheep Journal of Physiology $238371-386$

Greitz D and Hannerz J (1996) A proposed model of cerebrospinal fluid circulation observations with radionuclide cisternography American Journal of Neuroradiology $17431-438$

Hedlund L, Lischko MM, Rollag MD and Niswender GD (1977) Melatonin: daily cycle in plasma and cerebrospinal fluid of calves Science 195 686-687

Henry-Feugeas MC, Idy-Peretti I, Blanchet B, Hassine D, Zannoli G and Schouman-Claeys E (1993) Temporal and spatial assessment of normal cerebrospinal fluid dynamics with MR imaging Magnetic Resonance Imaging 11 1107-1118

Herkenham M (1987) Mismatches between neurotransmitter and receptor localizations in brain: observations and implications Neuroscience 23 1-38

Kanematsu N, Mori Y, Hayashi S and Hoshino K (1989) Presence of a distinct 24 -hour melatonin rhythm in the ventricular cerebrospinal fluid of the goat Journal of Pineal Research 7 143-152

Krueger JM, Fang I, Taishi P, Chen Z, Kushikata T and Gardi J (1998) Sleep. A physiologic role for IL-1 $\beta$ 
and TNF- $\alpha$ Annual New York Academy of Science $856148-159$

Lehman MN and Silver R (2000) CSF signaling in physiology and behaviour Progress in Brain Research $125415-432$

McComb JG (1983) Recent research into the nature of cerebrospinal fluid formation and absorption lournal of Neurosurgery 59 369-383

Malpaux B, Daveau A, Maurice-Mandon F, Duarte G and Chemineau P (1998) Evidence that melatonin acts in the premammillary hypothalamic area to control reproduction in the ewe: presence of binding sites and stimulation of luteinizing hormone secretion by in situ microimplant delivery Endocrinology 139 1508-1516

Møller M (1981) The ultrastructure of the deep pineal gland of the Mongolian gerbil and mouse: granular vesicle localization and innervation. In Pineal Function pp 257-266 Eds CD Matthews and RF Seamark. Amsterdam: Elsevier

Nicholson C (1999) Signals that go with the flow Trends in Neurosciences 22 143-145

Nilsson C, Lindvall-Axelsson M and Owman C (1992) Neuroendocrine regulatory mechanisms in the choroid plexus-cerebrospinal fluid system Brain Research Reviews 17 109-138

Pappenheimer JR, Miller TB and Goodrich CA (1967) Sleep-promoting effects of cerebrospinal fluid from sleep-deprived goats Proceedings National Academy of Science USA 58 513-517

Perlow MJ, Reppert SM, Boyar RM and Klein DC (1981) Daily rhythms in cortisol and melatonin in primate cerebrospinal fluid. Effects of constant light and dark Neuroendocrinology 32 193-196

Proescholdt MG, Hutto B, Brady LS and Herkenham $M$ (2000) Studies of cerebrospinal fluid flow and penetration into brain following lateral ventricle and cisterna magna injections of the tracer 114Clinulin in rat Neuroscience 95 577-592

Reppert SM, Perlow MJ, Tamarkin L and Klein DC (1979) A diurnal melatonin rhythm in primate cerebrospinal fluid Endocrinology 104 295-301

Rollag MD, Morgan RJ and Niswender GD (1978) Route of melatonin secretion in sheep Endocrinology 102 $1-8$

Schuster C, Gauer F, Malan A, Recio J, Pevet P and Masson-Pevet M (2001) The circadian clock, light: dark cycle and melatonin are differentially involved in the expression of daily and photoperiodic variations in $m t(1)$ melatonin receptors in the Siberian and Syrian hamsters Neuroendocrinology 74 55-68

Scott DE, Gash DM, Sladek IR, Clayton C, Mitchell JA, Calderon S and Paull WK (T982) Organization of the mammalian cerebral ventricular system: ultrastructural correlates of CSF - neuropeptide secretion Frontiers in Hormone Research 9 15-35

Shaw PF, Kennaway DJ and Seamark RF (1989) Evidence of high concentrations of melatonin in lateral ventricular cerebrospinal fluid of sheep Journal of Pineal Research 6 201-208.

Silver R, LeSauter J, Tresco PA and Lehman MN (1996) A diffusible coupling signal from the transplanted suprachiasmatic nucleus controlling circadian locomotor rhythms Nature 382 810-813

Skinner DC and Caraty A (2002) Measurement and possible function of $\mathrm{GnRH}$ in cerebrospinal fluid in ewes Reproduction Supplement 59 25-39

Skinner DC and Malpaux B (1999) High melatonin concentrations in third ventricular cerebrospinal fluid are not due to Galen vein blood recirculating through the choroid plexus Endocrinology 140 4399-4405

Speake T, Whitwell C, Kajita H, Majid A and Brown PD (2001) Mechanisms of CSF secretion by the choroid plexus Microscopy Research and Technique $\mathbf{5 2}$ 49-59

Strikic N, Klarica M, Vladic A and Bulat M (1994) Effect of active transport on distribution and concentration gradients of $[3 \mathrm{H}]$ benzylpenicillin in the cerebrospinal fluid Neuroscience Letters 169 159-162

Tortella FC and Long JB (1985) Endogenous anticonvulsant substance in rat cerebrospinal fluid after a generalized seizure Science 228 1106-1108

Tricoire $\mathrm{H}$, Locatelli $\mathrm{A}$, Chemineau $\mathrm{P}$ and Malpaux $\mathrm{B}$ (2002) Melatonin enters the cerebrospinal fluid through the pineal recess Endocrinology 143 84-90

Tricoire H, Malpaux B and Maller M (2003) Cellular lining of the sheep pineal recess studied by light, transmission- and scanning electron microscopy. Morphological indications for a direct secretion of melatonin from the pineal gland to the cerebrospinal fluid The Journal of Comparative Neurology 456 39-47

Vollrath L (1981) The pineal organ. In Handbuch der Mikroskopischen Anatomie des Menchen p 645 Eds A Oksche and L Vollrath. Springer-Verlag, Berlin

Withyachumnarnkul B and Knigge KM (1980) Melatonin concentration in cerebrospinal fluid, peripheral plasma and plasma of the confluens sinuum of the rat Neuroendocrinology $30382-388$

Wood JH (1982) Neuroendocrinology of cerebrospinal fluid: peptides, steroids and other hormones Neurosurgery 11 293-305

Zoli M, Torri C, Ferrari R, Jansson A, Zini I, Fuxe K and Agnati LF (1998) The emergence of the volume transmission concept Brain Research Review 26 136-147 